\title{
Dielectric Study on Cooperative Motion of Side Chain of Copoly $(\gamma$-methyl L-glutamate, $\gamma$-p-chlorobenzyl L-glutamate) in the Solid State
}

\author{
Shin YAgIHARA and Kunio HIKICHI \\ Department of Polymer Science, Faculty of Science, Hokkaido University, \\ Nishi 8-chome, Kita 10-jo, Kita-ku, Sapporo 060, Japan.
}

(Received November 7, 1980)

\begin{abstract}
Dielectric measurements on copoly $(\gamma$-methyl L-glutamate, $\gamma$ - $p$-chlorobenzyl Lglutamate) [copoly(MLG, $p$ ClBLG)] were performed in the frequency range of 0.03 to $300 \mathrm{kHz}$ and over the temperature range from -150 to $+130^{\circ} \mathrm{C}$ in order to study the side-chain motion. Two side-chain relaxations related respectively to MLG and $p$ CIBLG residues were observed. The results imply that there exists a cooperative motion of the side chains of poly ( $\alpha$-amino acid), though not as much as the main-chain motion of conventional amorphous polymers. The relaxation related to MLG residues is caused by clusters of MLG residues that are not affected by interactions between $p$ ClBLG residues.
\end{abstract}

KEY WORDS Dielectric Relaxation / Cooperative Motion / Side-Chain Motion / Copoly $(\gamma$-methyl L-glutamate, $\gamma$-p-chlorobenzyl L-glutamate) / Havriliak-Negami Equation / Free Volume / Dipole-Dipole Interaction /

A number of $\operatorname{poly}(\alpha$-amino acid)s take on an $\alpha$ helical form in the solid state. ${ }^{1}$ It is well known that side chains have great influence upon the conformation and physical properties of these polymers. ${ }^{2}$ Many studies ${ }^{2-11}$ have been made on the side-chain motion of poly ( $\alpha$-amino acid), and it has been pointed out that a large scale displacement of the whole side chain occurs cooperatively.

Dielectric $^{3-5}$ and mechanical ${ }^{2,6.7}$ measurements of poly $(\alpha$-amino acid $)$ s reveal a side-chain relaxation' caused by micro-Brownian motion throughout the side chain. It has also been reported that $\operatorname{copoly}(\gamma$-methyl L-glutamate, $\gamma$-benzyl L-glutamate)s[copoly(MLG, BLG)s] take on the $\alpha$-helical form in the solid state and show a single sidechain relaxation process brought about by a mechanism the same as that for the respective homopolymers. ${ }^{3}$ These facts support the proposition that the side-chain motion of poly $(\alpha$-amino acid) occurs cooperatively, and thus seems similar to the mainchain motion of conventional amorphous polymers. This was also confirmed by a dielectric study on $\operatorname{copoly}(\varepsilon-N$-benzyloxycarbonyl L-lysine, $\gamma$-benzyl Lglutamate). ${ }^{4}$ No other dielectric studies on the side- chain motion of $\operatorname{copoly}(\alpha$-amino acid $)$ have been reported.

It seems, however, that the side-chain motion of $\alpha$-helical poly $(\alpha$-amino acid) is less cooperative than that of the main-chain motion of a conventional amorphous polymer, since the scale of the molecular rearrangement for the side-chain motion of the poly $(\alpha$-amino acid $)$ is probably smaller than the main-chain motion of the conventional amorphous polymer. No evidence for this has as yet been provided experimentally. In the present work, we carried out dielectric measurements on copoly( $\gamma$-methyl L-glutamate, $\gamma$-p-chlorobenzyl L-giutamate)[copoly(MLG, $p$ ClBLG)] in order to clarify the cooperativity of the side-chain motion of poly $(\alpha$-amino acid $)$. The dielectric behavior of $\mathrm{PMLG}^{3}$ and $\mathrm{P} p \mathrm{ClBLG}{ }^{5}$ is comparatively different, since the $p$ ClBLG residue and its dipole moment are larger than those of the MLG residue. We examined the composition dependence of the dielectric behavior for these copolymers in detail. 


\section{EXPERIMENTAL}

Poly $(\gamma$-methyl L-glutamate) (PMLG) (Ajicoat A2000) was kindly provided by Ajinomoto Co. Copoly(MLG, $p$ ClBLG)s were prepared by the ester exchange reaction of PMLG with $p$ chlorobenzyl alcohol. ${ }^{12}$ The reaction was performed in 1,2-dichloroethane solution, and $p$-toluenesulfonic acid was used as a catalyst. The mole fraction of $p$ ClBLG $\phi_{\mathrm{p}}$ was determined from proton NMR spectra, ${ }^{12}$ and listed in Table I together with the sample code. The copolymer with the highest composition of $\phi_{\mathrm{p}}=0.99$ was approximately regarded as $\mathrm{P} p \mathrm{ClBLG}$.

Table I. Mole fractions of $p$ ClBLG for copoly(MLG, $p$ ClBLG)s

\begin{tabular}{lcc}
\hline \multicolumn{1}{c}{ Sample } & Code & $\begin{array}{c}\text { Mole fraction } \\
\text { of } p \text { ClBLG }\end{array}$ \\
\hline PMLG & - & 0 \\
Copoly & 1 & 0.06 \\
(MLG, $p$ ClBLG) & 2 & 0.12 \\
& 3 & 0.28 \\
& 4 & 0.35 \\
& 5 & 0.53 \\
P $p$ ClBLG & 6 & 0.75 \\
& 7 & 0.89 \\
& 8 & 0.94 \\
\hline
\end{tabular}

The dielectric constant $\varepsilon^{\prime}$ and the loss factor $\varepsilon^{\prime \prime}$ were measured using a TR-1C transformer bridge manufactured by Ando Denki Co. in a frequency range from 0.03 to $300 \mathrm{kHz}$ and over a temperature range from -150 to $+130^{\circ} \mathrm{C}$. A WBG-5 oscillator (Ando Denki Co.) and a Model LI-573 Lock-in amplifier (NF Circuit Design Block Co.) were used. Samples in the form of film each about $0.25 \mathrm{~mm}$ thick were cast from concentrated chloroform solutions and annealed at about $130^{\circ} \mathrm{C}$ for $48 \mathrm{~h}$. Silver was deposited in vacuo on both sides of the film to form the electrodes. Measurements were made in vacuo above room temperature and under a nitrogen atmosphere below room temperature.

$\mathrm{X}$-ray photographs were taken with a flat-film camera with nickel-filtered $\mathrm{Cu}-K_{\alpha}$ radiation. The sample-to-film distance was calibrated using the 111 reflection of Si powder coated on the surface of the sample.

\section{RESULTS}

Figure 1 shows the temperature dependence of the dielectric loss $\varepsilon^{\prime \prime}$ at a frequency of $1 \mathrm{kHz}$ for each copolymer. A small relaxation peak can be observed in the temperature vicinity of $-100^{\circ} \mathrm{C}$ for any one sample. On the basis of previous studies, ${ }^{3-6}$ this can be ascribed to the local motion of the side chain and is not considered further in this paper. All

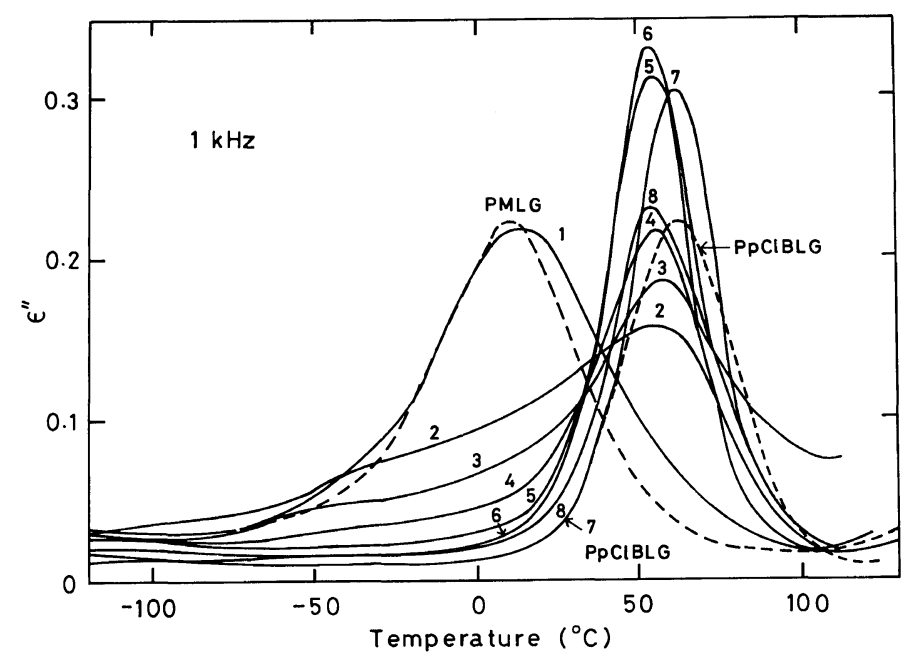

Figure 1. The temperature dependence of the dielectric loss $\varepsilon^{\prime \prime}$ at $1 \mathrm{kHz}$ for copoly (MLG, $\left.p \mathrm{ClBLG}\right) \mathrm{s}$. The number represents the copolymer code. 


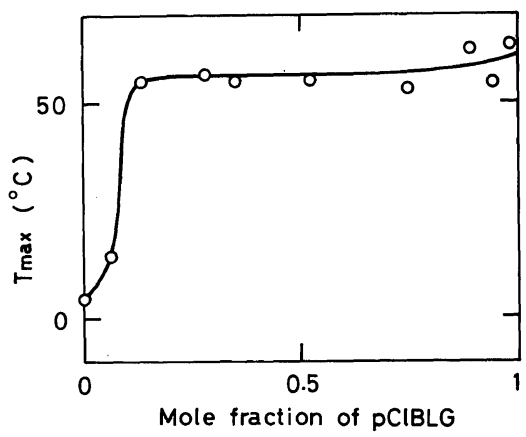

samples show large relaxation peaks above $0^{\circ} \mathrm{C}$, ascribable to the side-chain motion. ${ }^{3-6}$ Copolymer 1 shows such a peak in almost the same temperature range as does PMLG, while other copolymers show these peaks at higher temperatures. A detailed inspection of the relaxation peaks for copolymers 2 to 4 indicates that they are actually composed of

Figure 2. Composition dependence of the temperature of the maximum dielectric loss at a frequency of $1 \mathrm{kHz}$.

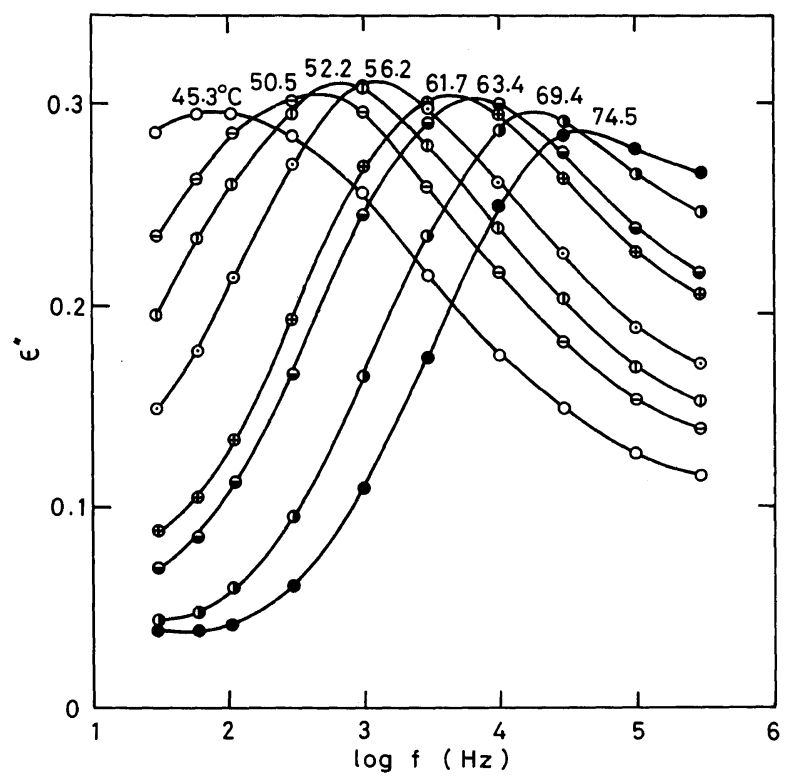

(a)

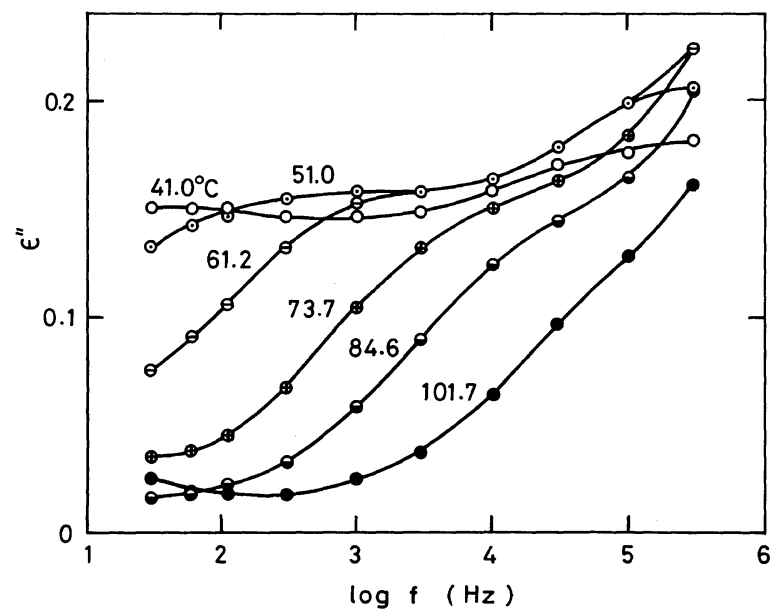

(b)

Figure 3. The frequency dependence of the dielectric loss $\varepsilon^{\prime \prime}$ at various temperatures: (a) for copolymer 5 , (b) for copolymer 2 . 


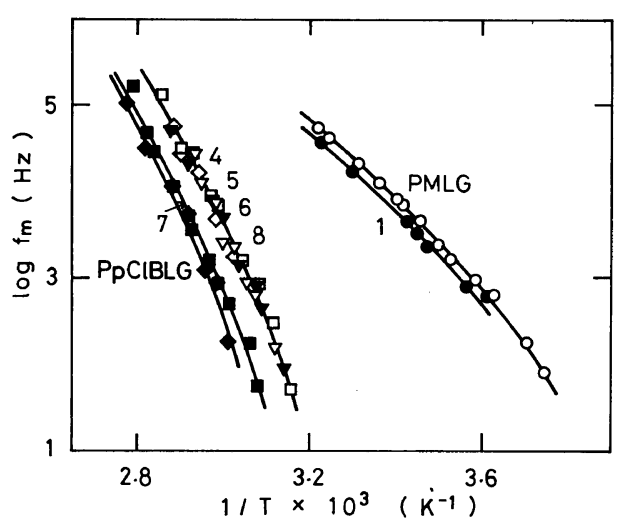

Figure 4. Plots of $\log f_{\mathrm{m}}$ against the reciprocal of the absolute temperature. two relaxation peaks. The one observed at lower temperatures becomes smaller in intensity and shifts to lower temperatures with increasing $\phi_{\mathrm{p}}$. This peak seems to vanish at $\phi_{\mathrm{p}} \simeq 0.5$. The other peak observed at higher tempratures is located at almost the same temperatures as that for P $p C l B L G$

The temperature $T_{\max }$ of the maximum dielectric loss at a frequency of $1 \mathrm{kHz}$ is plotted against $\phi_{\mathrm{p}}$ in Figure 2. The temperature $T_{\max }$ increases abruptly at $\phi_{\mathrm{p}} \simeq 0.1$ with increasing $\phi_{\mathrm{p}}$ and tends to be constant in the region $\phi_{\mathrm{p}}>0.1$.

The frequency dependence of $\varepsilon^{\prime \prime}$ is shown for copolymer $5\left(\phi_{\mathrm{p}}=0.53\right)$ and copolymer $2\left(\phi_{\mathrm{p}}=0.12\right)$ at various temperatures near $T_{\max }$ in Figures $3 \mathrm{a}$ and $3 b$, respectively.

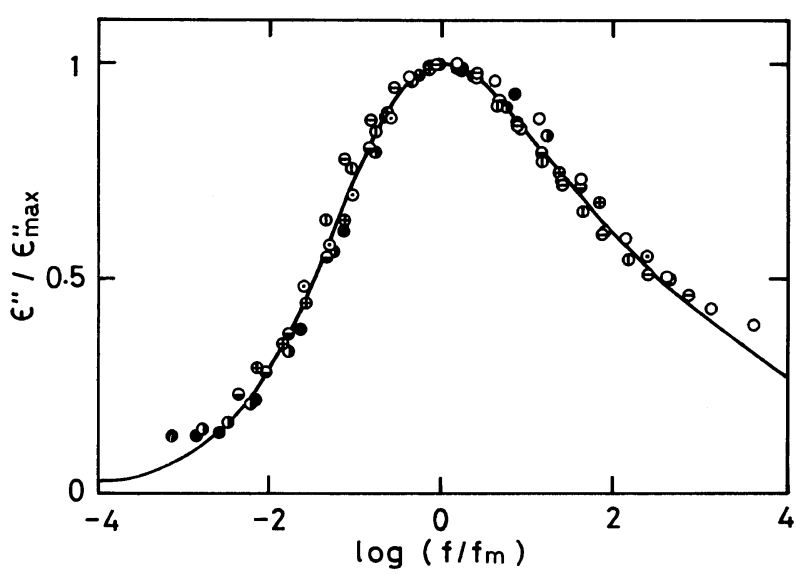

Figure 5. Normalized loss curve for copolymer 5. Solid line is obtainæd from eq 1, using the parameters $\alpha=0.40$ and $\beta=0.28$. (○) $45.3^{\circ} \mathrm{C} ;(\ominus) 50.5 ;(1) 52.5 ;(\odot) 56.2 ;(\oplus) 61.7 ;(\ominus) 63.4 ;(\bigcirc) 69.4 ;(\bigcirc) 74.5$.

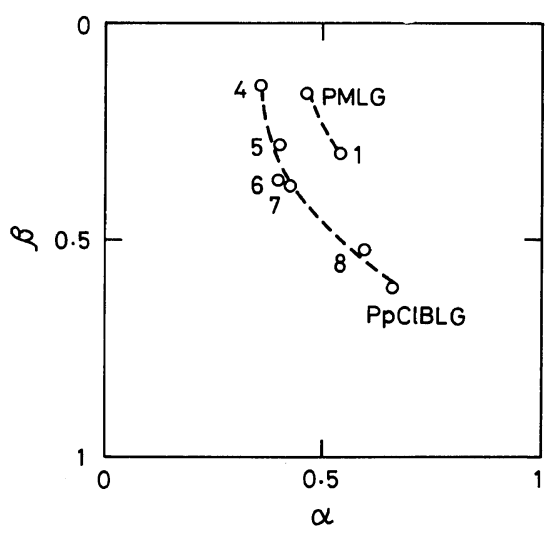

Figure 6. Value of parameters $\alpha$ and $\beta$ for each copolymer. Number represents the copolymer code.
The logarithm of the frequency of the maximum dielectric loss, $\log f_{\mathrm{m}}$, is plotted against reciprocal absolute temperature in Figure 4. We could not obtain the $f_{\mathrm{m}}$ for copolymers 2 and 3 , since determination of their relaxation peaks involves ambiguity due to the double-peak structure of the relaxation shown in Figure $3 \mathrm{~b}$. The apparent activation energy can be obtained from the slope of the line in Figure 4, being about $30 \mathrm{kcal} / \mathrm{mol}$ for copolymers with $\phi_{\mathrm{p}}<0.1$ and $50 \mathrm{kcal} / \mathrm{mol}$ for those with $\phi_{\mathrm{p}}>0.3$, both at $1 \mathrm{kHz}$.

The normalized loss curve obtained from the frequency dependence of $\varepsilon^{\prime \prime}$ can be expressed in terms of Havriliak-Negami equation, ${ }^{13}$

$$
\frac{\varepsilon^{*}-\varepsilon_{\infty}{ }^{\prime}}{\varepsilon_{0}{ }^{\prime}-\varepsilon_{\infty}{ }^{\prime}}=\left[1+(i \omega \tau)^{1-\alpha}\right]^{-\beta},
$$


where $\varepsilon^{*}$ is the complex dielectric constant $\varepsilon^{*}=\varepsilon^{\prime}-i \varepsilon^{\prime \prime}, \varepsilon_{0}^{\prime}$ and $\varepsilon_{\infty}{ }^{\prime}$ are the low- and highfrequency limits of the dielectric constant, respectively; $\omega$ is the angular frequency, $\tau$ is the relaxation time, and $\alpha$ and $\beta$ are parameters characterizing the shape of the absorption curve. The normalized loss curve for copolymer $5\left(\phi_{\mathrm{p}}=0.53\right)$ is shown in Figure 5 . The change in shape of the dielectric loss curve with composition is represented by the $\alpha-\beta$ diagram in Figure 6. A discontinuity appears between copolymers with $\phi_{\mathrm{p}}<0.1$ and those with $\phi_{\mathrm{p}}>0.3$.

\section{DISCUSSION}

The results obtained above indicate that copolymers with $\phi_{\mathrm{p}}<0.1$ and $\phi_{\mathrm{p}}>0.3$ have dielectric properties similar to those of PMLG and P $p$ ClBLG, respectively. It seems that the interaction between MLG residues is dominant for cocpolymers with $\phi_{\mathrm{p}}<0.1$, while the interaction between $p$ ClBLG residues is dominant for those with $\phi_{\mathrm{p}}>0.3$. Therefore, it is likely that one of the two relaxations observed in copolymers 2 to 4 at higher temperatures is related primarily to $p$ ClBLG residues, and the other observed at lower temperatures is related to MLG residues. Figure 1 shows that the magnitude of the relaxation related to $p$ ClBLG residues is obviously larger than that related to the MLG residues, though the mole fraction of $p$ ClBLG is smaller than that of the MLG residues. This fact indicates that the relaxation related to MLG residues can be attributed to clusters larger than the critical size and not be affected by $p$ ClBLG residues. It seems that these clusters begin to decrease in number suddenly at $\phi_{\mathrm{p}} \simeq 0.1$ and vanish at $\phi_{\mathrm{p}} \simeq 0.5$.

The double-peak structure has not been reported so far for side-chain relaxation of $\operatorname{copoly}(\alpha$-amino

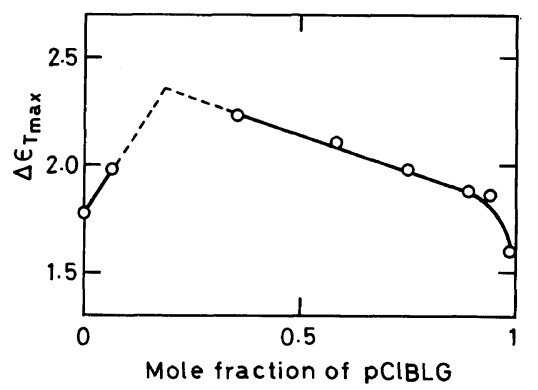

Figure 7. Composition dependence of the magnitude of the dielectric relaxation. acid). In the case of conventional amorphous polymers, a single relaxation due to a cooperative main-chain motion ${ }^{14}$ and relaxations due to nocooperative local motions of respective monomer units $^{15}$ are observed. Thus, previous investigations $^{3,4}$ indicate that there exists a cooperativity in the side-chain motions of $\operatorname{poly}(\alpha$-amino acid) in the $\alpha$-helical form. However, the double-peak structure observed in the present work suggests that this cooperativity is not so large as that in the mainchain motion of conventional amorphous polymers.

The distribution of MLG and BLG residues along the chain was determined from the NMR spectra for copoly(MLG, BLG). ${ }^{16}$ In the present case, NMR spectra did not provide any information on the distribution of $p$ ClBLG residues along the chain. Konishi and Hatano ${ }^{17}$ reported that, in the process of the ester exchange reaction of PMDG with $p$-chlorobenyl alcohol, a $p$ ClBDG residue shields a neighboring $0.5 \mathrm{MDG}$ residue of a copolymer with $0<\phi_{\mathrm{p}}<0.5$, and that units constituting the $p$ ClBDG residue and neighboring 0.5 MDG residue are randomly distributed in the chain. Accordingly, the distribution of MLG and $p$ ClBLG residues in the present copolymers with $0<\phi_{\mathrm{p}}<0.5$ is assumed to be almost random.

The magnitude of the dielectric relaxation, $\Delta \varepsilon=\varepsilon_{0}{ }^{\prime}-\varepsilon_{\infty}{ }^{\prime}$, was calculated from eq 1 at the temperature $T_{\max }$ for each copolymer and is plotted against $\phi_{\mathrm{p}}$ in Figure 7. A maximum appears in the region $0.2<\phi_{\mathrm{p}}<0.3$. These results will be discussed later.

The composition dependence of the X-ray diffraction pattern of the copolymers was studied. All copolymers showed an intense reflection corre-

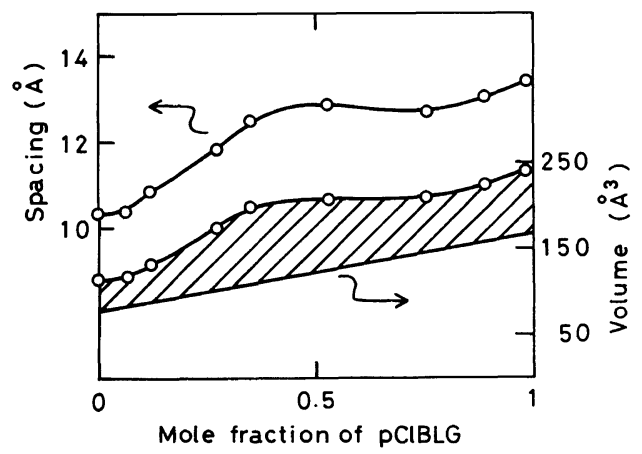

Figure 8. Composition dependence of spacing and volume. 
sponding to the $11-13 \AA$ spacing. This spacing is related to the inter-helix distance in the crystalline phase, where helical molecules are packed parallel to each other. ${ }^{1}$ Figure 8 shows the spacing as a function of $\phi_{\mathrm{p}}$. It is seem that the spacing increases gradually with increasing $\phi_{\mathrm{p}}$ and reaches a constant value at $\phi_{\mathrm{p}} \simeq 0.5$. No abrupt change in the spacing was detected in a composition range where the dielectric properties changed suddenly.

It has been reported that PMLG $^{1}$ and $\mathrm{P} p \mathrm{ClBLG}^{18}$ in the helical form are packed in a hexagonal array. It is believed that the copolymers take on the $\alpha$-helical form and then become packed in the hexagonal array. Going on the assumption of this hexagonal packing, the average volume per side chain was calculated from the spacing and is plotted in Figure 8. The van der Waals volume per side chain in each copolymer was estimated using values of respective groups reported in the literature ${ }^{19}$ on the assumption of additivity. The difference between the measured and calculated volumes is the free volume $e^{20}$ and is represented by a shaded area in Figure 8 . The ratio of the free volume to the total volume, i.e., the fractional free-volume, exhibits a maximum at $\phi_{\mathrm{p}} \simeq 0.3$. These results are similar to those for the magnitude of relaxation. Such similarity suggests that the appearance of a maximum in $\Delta \varepsilon$ at $0.2<\phi_{\mathrm{p}}<0.3$ reflects an increase in the effective dipole moment caused by a decrease in the steric hindrance resulting from an increase in free volume. The appearance of a maximum in the fractional free-volume also explains the shift of the small relaxations of copolymers 2 to 4 to lower temperatures. However, the steep increase in the temperature $T_{\max }$ at $\phi_{\mathrm{p}} \simeq 0.1$ cannot be explained by the increase in the free-volume fraction. This means that the composition dependence of the side-chain relaxations of these copolymers cannot be interpreted on the basis of free volume only. A similar disagreement has also been reported for copoly(MLG, BLG). ${ }^{3}$ For the present copolymers this disagreement is more noticeable and probably arises from the large dipole-dipole interaction between $p$ ClBLG residues. The dipole-dipole interaction must be taken into account to describe the sidechain relaxation of poly $(\alpha$-amino acid).

\section{CONCLUSION}

A double-peak structure was observed for the dielectric relaxations of copoly(MLG, $p$ ClBLG)s in the range $0.1<\phi_{\mathrm{p}}<0.5$. The relaxation observed at higher temperatures was determined by the interaction between the $p$ ClBLG residues, and the other was determined by the interaction between the MLG residues. The latter was ascribed to the clusters of MLG residues which were not influenced by the interaction between $p$ ClBLG residues. It was concluded that there is a cooperativity in the sidechain motion of poly $(\alpha$-amino acid), though not so much as the main-chain motion of conventional amorphous polymers. The composition dependence of the magnitude of the relaxation was interpreted on the basis of free volume. However, the dipoledipole interaction must be taken into account to exaplain in detail the dielectric properties of $\operatorname{poly}(\alpha-$ amino acid).

Acknowledgment. The authors wish to thank Dr. T. Hiraoki for NMR measurement. This work was supported by the Ministry of Education, Science, and Culture of Japan.

\section{REFERENCES}

1. A. Elliott, "Poly- $\alpha$-Amino Acids," Ed., G. D Fasman, Marcel Dekker, New York, N.Y., 1967, Chapter 1.

2. A. Hiltner, J. M. Anderson, and E. Bolkowski, Macromolecules, 5, 446 (1972).

3. A. Tsutsumi, K. Hikichi, T. Takahashi, Y. Yamashita, N. Matsushima, M. Kanke, and M. Kaneko, J. Macromol. Sci., Phys., B8, 413 (1973).

4. N. Sasaki, Y. Yamashita, A. Tsutsumi, and K. Hikichi, Polym. J., 10, 207 (1978).

5. N. Sasaki, A. Tsutsumi, K. Hikichi, Y. Konishi, and M. Hatano, Polym. J., 11, 583 (1979).

6. R. G. Saba, J. A. Sauer, and A. E. Woodward, $J$. Polym. Sci., A, 1, 1483 (1963).

7. T. Kajiyama, M. Kuroishi, and M. Takayanagi, $J$. Macromol. Sci., Phys., B11, 121 (1975).

8. L. A. E. Kail, J. A. Sauer, and A. E. Woodward, $J$. Phys. Chem., 66, 1292 (1962).

9. K. Hikichi, J. Phys. Soc. Jpn., 19, 2169 (1964).

10. A. J. McKinnon and A. V. Tobolski, J. Phys. Chem., 70, 1453 (1966).

11. K. Hikichi, A. Tsutsumi, S. Isozaki, and M. Kaneko, Polym. J., 7, 646 (1975).

12. E. M. Bradbury, B. G. Carpenter, and H. Goldman, Biopolymers, 6, 837 (1968).

13. S. Havriliak and S. Negami, J. Polym. Sci., C, 14, 99 (1966).

14. N. G. McCrum, B. E. Read, and G. Williams, "Anelastic and Dielectric Effects in Polymeric 
Solids," Wiley, New York, N.Y., 1969, Chapters $8-11$.

15. Y. Kawamura, S. Nagai, J. Hirose, and Y. Wada, $J$. Polym. Sci., A-2, 7, 1559 (1969).

16. T. Hiraoki, A. Tsutsumi, K. Hikichi, and $M$. Kaneko, Polym. J., 8, 524 (1976).

17. Y. Konishi and M. Hatano, J. Polym. Sci., Polym. Chem. Ed., 14, 2329 (1976).
18. M. Osanai and K. Hikichi, Biopolymers, 19, 1099 (1980).

19. G. L. Slonimskii, A. A. Askadskii, and A. I. Kitaigorodskii, Vysokomol. Soedin., Ser. A, 12, 494 (1970).

20. A. K. Doolittle, J. Appl. Phys., 22, 1471 (1951); ibid., 23, 236 (1952). 